\title{
Adaptive Estimation of Time-varying Parameters with Application to Roto-magnet Plant
}

\author{
Jing Na, Member, IEEE, Yashan Xing, and Ramon Costa-Castelló, Senior Member, IEEE
}

\begin{abstract}
This paper presents an alternative adaptive parameter estimation framework for nonlinear systems with time-varying parameters. Unlike existing techniques that rely on the polynomial approximation of time-varying parameters, the proposed method can directly estimate the unknown time-varying parameters. Moreover, this paper proposes several new adaptive laws driven by the derived information of parameter estimation errors, which achieve faster convergence rate than conventional gradient descent algorithms. In particular, the exponential error convergence can be rigorously proved under the well-recognized persistent excitation (PE) condition. The robustness of the developed adaptive estimation schemes against bounded disturbances is also studied. Comparative simulation results reveal that the proposed approaches can achieve better performance than several conventional estimation algorithms. Finally, the proposed parameter estimation methods are verified by conducting experiments based on a roto-magnet plant.

Index Terms - Adaptive parameter estimation, time-varying parameters, nonlinear systems.
\end{abstract}

\section{INTRODUCTION}

In most control designs, it is presumed that precise model of the system to be controlled should be known, which stimulated the study of system identification and parameter estimation. During the past decades, extensive researches have been carried out on system identification and adaptive parameter estimation [1]-[3]. It is known that early work on adaptive parameter estimation has been developed based on the gradient descent algorithms [3], least-squares (LS) or recursive least-squares (RLS) methods [1]. In this framework, the unknown parameters are online estimated by minimizing the predictor/observer output error. Although the convergence of estimation error can be verified under the condition that the regressor vector or matrix satisfies the persistent excitation (PE) condition [1], the

This work was supported by the National Natural Science Foundation of China (NSFC) under grant 61573174, the Spanish Ministerio de Educación project DPI2015-69286-C3-2-R (MINECO/ FEDER), the Spanish State Research Agency through the María de Maeztu Seal of Excellence to IRI (MDM-2016-0656), the AGAUR of Generalitat de Catalunya through the Advanced Control Systems (SAC) group grant (2017 SGR 482), and the Chinese Scholarship Council (CSC) under rant 201808390007.

$\mathrm{J}$. Na is with the Faculty of Mechanical \& Electrical Engineering, Kunming University of Science \& Technology, Kunming, 650500, P.R. China (Email: najing25@163.com).

Y. Xing is with the Faculty of Mechanical and Electrical Engineering, Kunming University of Science and Technology, Kunming 650500, China, and also the Institut de Robòtica i Informàtica Industrial, CSIC-UPC, Llorens i Artigas 4-6, 08028 Barcelona, Spain (e-mail: yashan.xing@upc.edu).

R. Costa-Castelló is with the Institut de Robòtica i Informàtica Industrial, CSIC-UPC, Llorens i Artigas 4-6, 08028 Barcelona, Spain (e-mail: ramon.costa@upc.edu). robustness of these algorithms against noise and disturbances has been criticized in the literature [4]. In the subsequent developments, the robustness of these algorithms was improved by introducing several modifications in the adaptive laws, i.e., $e$-modification, $\sigma$-modification, and dead-zone modification [5]. However, with these modifications, the parameter estimation error can converge to a residual set rather than zero, i.e., the convergence of the estimated parameters to their true values cannot be retained. Moreover, it is also well-recognized that the adaptive estimation methods based on the gradient algorithms are mainly feasible for the cases where the parameters to be estimated are constant. Hence, these estimation methods may fail to retain satisfactory performance for time-varying parameters [6], [7]. However, in practical systems, time-varying parameters may be unavoidable [7], [8], which cannot be ignored in the system modeling.

In fact, the online estimation of time-varying parameters has remained as a theoretically challenging, yet practically useful, open problem. On the other hand, the limitations of classical adaptive estimation algorithms have also stimulated several studies on investigating advanced adaptive estimation algorithms for time-varying parameters. On this topic, there have been two major ideas: 1) transform the systems with time-varying parameters to alternative systems with constant parameters and then use the gradient based algorithms [9]-[11]; 2) exploit the ability of gradient based algorithms and further tailor them for time-varying parameters [12]-[15].

In order to use existing estimation schemes developed for constant parameters, several polynomials have been used to locally approximate time-varying parameters in the system. In particular, the time-varying parameters can be approximated by time-related polynomials with unknown constant coefficients [9], which have been obtained based on a local Taylor's series expansion. Hence, the unknown constant coefficients in the Taylor series can be estimated by the LS methods in [9]. In [10], the time-varying parameters were approximated by a novel polynomial, and then the LS method was further adopted to estimate the associated unknown coefficients. In [11], the unknown constant coefficients of the used polynomials were estimated by using a novel adaptive law driven by the parameter estimation error (the difference between the unknown parameters and their estimates). However, the disturbances and the residual approximation errors may make the convergence of the estimated parameter sluggish. Nevertheless, the use of polynomials leads to an indirect, two-step estimation procedure, and the required resetting schemes also result in the increased complexity and heavy computational costs in the practical implementation.

To avoid using polynomial approximation, some works have 
also been carried out to explore the ability of conventional adaptive estimation schemes. In [12], the gradient algorithm with a project operation was used to estimate time-varying parameters assuming the knowledge of the upper bounds of unknown parameters is known. In [13], a stochastic gradient method was adopted to estimate time-varying parameters for dual-rate systems. In [14], the gradient methods, the recursive and non-recursive LS approaches with/without a forgetting factor were compared in details. As it is stated in [14], the non-recursive LS algorithms can retain robustness against bounded disturbances. Lozeno et al. [15] proposed a modified LS algorithm with $\sigma$-modification to address linear time-varying systems with bounded disturbances. These methods all require the upper bounds of the time-varying parameters and their derivatives, so that they can be used for slowly varying parameters only with a priori known knowledge. Recently, a new set-based estimation method was investigated for systems with time-varying parameters [7], where a novel adaptive law and an uncertainty set updating scheme were all introduced. Ding et al. [16] proposed a modified project identification method and proved its convergence based on the stochastic theory. In [17], an almost invariant manifold approach was used to design an adaptive law to estimate the unknown time-varying parameters. Yang et al. [18] and Guo et al. [19] applied the expectation maximization algorithm to estimate the unknown parameters for process systems with missing data. These above-mentioned methods can prove the convergence of the estimation error, while the invertibility of the regressor has to be validated online. Moreover, an extra observer/predictor should be constructed to derive the adaptive laws, which also leads to the increased computational costs.

To the best of our knowledge, online adaptive estimation of time-varying parameters has not been fully solved in the literature. Motivated by this fact, a novel adaptive estimation approach is introduced for time-varying parameters. The basic idea is to further tailor our recent work [20]-[22], which reported a new parameter estimation framework for constant parameters. Different to above-mentioned gradient or LS based algorithms, the proposed adaptive laws are designed by using the extracted parameter estimation errors. Hence, a set of intermediate variables are first derived by applying simple low-pass filters on the available system dynamics. Then the difference between the unknown parameters and their estimates can be calculated and then adopted to design adaptive laws for parameter estimation, which can guarantee the exponential estimation error convergence. Moreover, the design and use of time-varying learning gains are also investigated to eliminate the effects of the regressor dynamics on the convergence speed. Comparisons to several available algorithms are provided. This new adaptive estimation does not need to measure the derivatives of system states and design any observer/predictor. Especially, the online validation of the invertibility of the regressor matrix and the computation of matrix inverses (if feasible) can be avoided. Comparative simulations between the proposed methods and the gradient and LS methods illustrate that this new estimation approach can accomplish better performance. Finally, the algorithms are also practically verified using an educational roto-magnet plant built in our lab.

The paper is structured as follows: Section II describes the problem formulation. The adaptive laws with exponential convergence are proposed in Section III. Section IV presents the comparisons to other estimation approaches. Simulations are provided in Section V, and experimental results are shown in Section VI. Conclusions are outlined in Section VII.

\section{PROBLEM FORMULATION}

This paper studies the following nonlinear system with time-varying parameters

$$
\dot{x}=F(x, u)+R(x, u) \alpha(t)+d(t)
$$

where $x \in \mathbb{R}^{n}$ is the system states vector; $u \in \mathbb{R}^{m}$ is the system input; $\alpha(t)=\left[\alpha_{1}(t), \alpha_{2}(t), \cdots, \alpha_{p}(t)\right]^{T} \in \mathbb{R}^{p}$ defines the unknown time-varying parameters to be estimated; $F(x, u) \in \mathbb{R}^{n}$ is a known nonlinear function, and $R(x, u) \in \mathbb{R}^{n \times p}$ is a known regressor matrix; $d(t) \in \mathbb{R}^{n}$ denotes the effects of external disturbances and noise.

The problem to be addressed is to estimate the time-varying parameter vector $\alpha(t)$ by using available system input $u$ and states $x$. It is noted that the time-varying property of the parameters makes the parameter estimation more difficult than the cases with constant parameters since the well-known parameter estimation methods (e.g., gradient [1], [5] or RLS [1]) are valid for system (1) only if the unknown parameters in $\alpha(t)$ are constant or slowly varying. Moreover, an observer or predictor must be designed in these schemes, and thus the convergence speed of the estimation error is also difficult to evaluate. Hence, these methods have limited ability to estimate fast time-varying parameters [14]. On the other hand, the indirect estimation schemes with polynomial approximation, e.g., [9]-[11], require heavy computational costs due to the use of time-dependent polynomials and the resetting mechanisms. These facts motivate us to develop a simple, efficient adaptive estimation framework to estimate time-varying parameters in $\alpha(t)$ without using any observer and polynomial approximation, whilst the convergence can be guaranteed.

The following well-recognized assumptions to be used in the analysis are summarized as follow:

Assumption 1: The states $x$ and input $u$ of system (1) are all measurable and bounded. The unknown disturbance $d$ is bounded. Moreover, $R(x, u)$ and $F(x, u)$ are continuous functions of $x$ and $u$, which are also bounded.

Assumption 2: The time derivative of the unknown parameter vector $\alpha(t)$ is bounded, i.e., $\|\dot{\alpha}\| \leq \varpi$ is true for a positive constant $\varpi>0$.

Remark 1: Assumption 1 and Assumption 2 have been widely used in the parameter estimation literatures [10], [14], [15], and they can be fulfilled in most practical applications. Specifically, the assumptions are less stringent than those used in [10], [15], where a priori knowledge on the upper bounds of the unknown time-varying parameters are required. In this paper, the upper 
bound parameter $\varpi$ in Assumption 2 is used for analysis only, and thus its true value is not necessarily known.

\section{TIME-VARYING PARAMETER ESTIMATION}

This section will introduce a novel adaptive law to estimate $\alpha(t)$ in system (1) to achieve exponential convergence. Inspired by our previous work [20], [21], which has been initially proposed for constant parameters, the filtered variables $x_{f}, F_{f}$ and $R_{f}$ of $x, F$ and $R$ are defined as

$$
\begin{cases}k \dot{x}_{f}+x_{f}=x, & x_{f}(0)=0 \\ k \dot{F}_{f}+F_{f}=F, & F_{f}(0)=0 \\ k \dot{R}_{f}+R_{f}=R, & R_{f}(0)=0\end{cases}
$$

where $k>0$ is the filter coefficient, which can be set as a small constant. According to Assumption 1, $R_{f}$ is bounded by $\left\|R_{f}\right\| \leq \mu$ for a positive scalar $\mu>0$ since $R_{f}$ is the filtered version of $R$.

By filtering both sides of system (1) with the low-pass filter given in (2), we can obtain that

$$
\frac{s}{k s+1}[x]=\frac{1}{k s+1}[F]+\frac{1}{k s+1}[R \alpha]+\frac{1}{k s+1}[d]
$$

Considered the equations (2) - (3) and Swapping Lemma [5] regarding the term $\frac{1}{k s+1}[R \alpha(t)]$, it can be verified that

$$
\begin{aligned}
\dot{x}_{f} & =\frac{x-x_{f}}{k}=F_{f}+R_{f} \alpha-\frac{k}{k s+1}\left[R_{f} \dot{\alpha}\right]+d_{f} \\
& =F_{f}+R_{f} \alpha+\zeta
\end{aligned}
$$

where $d_{f}$ given by $k \dot{d}_{f}+d_{f}=d, d_{f}(0)=0$ is the filtered disturbance, which is bounded. Since $R_{f}$ is bounded, then according to Assumption 2, the term $\zeta=(k /[k s+1])\left[R_{f} \dot{\alpha}\right]-d_{f}$ is also bounded for any constant $k>0$, i.e., there exists a constant $\gamma>0$, such that the fact $\|\zeta\| \leq \gamma$ holds. Hence, the term $\zeta$ could be taken as a bounded disturbance in system (4). Then, the following lemma can be obtained:

Lemma 1: By applying the filter operation (2) on system (1), then the intermediate variable

$$
\beta=\frac{x-x_{f}}{k}-F_{f}-R_{f} \alpha
$$

is ultimately bounded and decreases exponentially to a set around zero. Specifically, for $d=0$ we have

$$
\lim _{k \rightarrow 0}\left\{\lim _{t \rightarrow \infty}\left[\left(x-x_{f}\right) / k-F_{f}-R_{f} \alpha\right]\right\}=0
$$

Thus, for any low-pass filter coefficient $k>0$ and $d=0$, the surface $\left(x-x_{f}\right) / k-F_{f}-R_{f} \alpha=0$ is an invariant manifold [23].

Proof: We can calculate the differential of $\beta$ with respect to time from (2) and (5) as

$$
\dot{\beta}=\frac{\dot{x}-\dot{x}_{f}}{k}-\dot{F}_{f}-\dot{R}_{f} \alpha-R_{f} \dot{\alpha}=-\frac{1}{k}\left(\beta+k R_{f} \dot{\alpha}\right)
$$

We first prove that $\beta$ is bounded. By choosing Lyapunov function $V_{\beta}=\beta^{T} \beta / 2$, we can obtain $\dot{V}_{\beta}$ along (7) as

$$
\begin{aligned}
\dot{V}_{\beta} & =-\frac{1}{k} \beta^{T} \beta-\beta^{T} R_{f} \dot{\alpha} \leq-\frac{1}{k}\|\beta\|^{2}+\frac{1}{2 k}\|\beta\|^{2}+\frac{k}{2}\left\|R_{f} \dot{\alpha}\right\|^{2} \\
& \leq-\frac{1}{k} V_{\beta}+\frac{k}{2} \mu^{2} \varpi^{2}
\end{aligned}
$$

The above inequality indicates that $V_{\beta} \leq e^{-t / k} V_{\beta}(0)+k^{2} \mu^{2} \varpi^{2} / 2$. Thus, $\beta$ is bounded and decreases based on Lyapunov theory. Specifically, we can verify that $\beta(t)$ will converge to a set defined by $\|\beta(t)\|=\sqrt{2 V_{\beta}(t)} \leq \sqrt{\beta^{2}(0) e^{-t / k}+k^{2} \mu^{2} \varpi^{2}}$, of which the ultimate size is affected by the filter coefficient $k$, the upper bound $\mu$ of $R_{f}$ and the upper bound $\varpi$ of $\dot{\alpha}$. Hence, we can verify that $\lim _{t \rightarrow \infty} \beta(t)=k \mu \varpi$ holds, such that $\beta(t)$ is vanishing for an adequately small $k$ or when the parameters are constant (i.e., $\varpi=0$ ), i.e., $\lim _{k \rightarrow 0} \lim _{t \rightarrow \infty} \beta(t)=0$ as $k \rightarrow 0$ for any bounded initial condition $\beta(0)$. Hence, the surface $\beta=0$ is an invariant manifold [23] with a positive constant $k>0$. This completes the proof.

Remark 2: As shown in (4) and the proof of Lemma 1, the filter constant $k$ in (2) determines the ultimate residual error bounds of $\zeta$ (i.e., $\|\zeta\| \leq \gamma$ ) and $\beta$. This stems from the use of Swapping Lemma on the time-varying parameters, which leads to the residual term $\zeta$. Thus, this filter constant $k$ should be set as a small constant in general to obtain accurate parameter estimation. On the other hand, the constant $k$ defines the bandwidth of the low-pass filter $1 /(k s+1)$, which is related to robustness against noise and disturbance. Hence, the filter constant $k$ should be set to make a tradeoff between the convergence speed and robustness.

It is shown that the manifold variable (5) does not depend on the derivative of state vector $\dot{x}$. Additionally, it provides an implicit relationship between the unknown parameters $\alpha$ and the available variables $\left(x, x_{f}, F_{f}, R_{f}\right)$. Hence, we can design an adaptive law based on the merit of this invariant manifold. For this purpose, an intermediate regressor matrix $G$ and an intermediate vector $S$ are defined as

$$
\begin{cases}\dot{G}=-\delta G+R_{f}^{T} R_{f}, & G(0)=0 \\ \dot{S}=-\delta S+R_{f}^{T}\left[\left(x-x_{f}\right) / k-F_{f}\right], & S(0)=0\end{cases}
$$

where $\delta>0$ is a constant, which serves as a forgetting factor to guarantee the boundedness of matrix $G$ and vector $S$.

To design an adaptive law by using regressor $G$ in (9), the positive definiteness of this matrix needs to be analyzed. Denote $\lambda_{\max }(\cdot), \lambda_{\min }(\cdot)$ as the maximum and minimum matrix eigenvalues, we can obtain:

Lemma 2 [20], [21]: If the original regressor matrix $R$ is PE (e.g., there exist $T>0, \varepsilon>0$ such that $\int_{t}^{t+T} R(\tau) R^{T}(\tau) d \tau \geq \varepsilon I, \forall t \geq 0$ ), then the matrix $G$ defined in (9) is positive definite, that is $\lambda_{\min }(G)>\sigma_{1}>0$ for positive constant $\sigma_{1}$.

Proof: A similar proof of the above lemma can be found in [20], [21], and thus will not be presented here.

Based on the derived $G(t), S(t)$ given in (9), other auxiliary 
vectors $P_{1}(t)$ and $P_{2}(t)$ used to design the adaptive laws can be calculated as

$$
\begin{gathered}
P_{1}(t)=G(t) \hat{\alpha}(t)-S(t) \\
P_{2}(t)=R_{f}^{T} R_{f} \hat{\alpha}(t)-R_{f}^{T}\left[\left(x-x_{f}\right) / k-F_{f}\right]
\end{gathered}
$$

where $\hat{\alpha}(t)$ is the estimate of the unknown parameters $\alpha(t)$, which is online calculated based on the adaptive laws to be proposed in the following (15) and (20).

Now, we have the following fact:

Lemma 3: For the variable given in (10) and (11), we can verify that

$$
\begin{gathered}
P_{1}(t)=-G(t) \tilde{\alpha}(t)+\psi \\
P_{2}(t)=-R_{f}^{T} R_{f} \tilde{\alpha}(t)+R_{f}^{T} \zeta
\end{gathered}
$$

where $\tilde{\alpha}=\alpha-\hat{\alpha}$ is the estimation error, $\psi=\int_{0}^{t} e^{-\delta(t-\tau)} R_{f}^{T}(\tau) \zeta(\tau) d \tau$ is a residual variable stemming from the variation of unknown parameters $\alpha(t)$, which is bounded by $\|\psi\| \leq\left\|R_{f}\right\|\|\zeta\| / \delta=\mu \gamma / \delta$. Proof: To prove equation (12), the solution of (9) can be derived as

$$
\left\{\begin{array}{l}
G(t)=\int_{0}^{t} e^{-\delta(t-\tau)} R_{f}^{T}(\tau) R_{f}(\tau) d \tau \\
S(t)=\int_{0}^{t} e^{-\delta(t-\tau)} R_{f}^{T}(\tau)\left[\left(x(\tau)-x_{f}(\tau)\right) / k-F_{f}(\tau)\right] d \tau
\end{array}\right.
$$

Then we can verify from (4) that $S(t)=G(t) \alpha(t)-\psi$, which together with (10) can lead to (12). The proof of (13) can be carried out by substituting (4) into (11).

Remark 3: It is shown in Lemma 3 that the estimation error $\tilde{\alpha}$ is involved in the obtained variables $P_{1}, P_{2}$. In particular, $P_{1}$ is a filtered version of $P_{2}$ in terms of a low pass filter $1 /(s+\delta)$, which introduces an 'averaging' effect on the regressor $R_{f}^{T} R_{f}$, and thus helps improving the robustness against noise. However, this averaging operation could decrease the ability to track fast varying parameters. Hence, the use of $P_{2}$ with the instant information of estimation error $\tilde{\alpha}$ is essential for tracking unknown time-varying parameters in comparison to the case with constant parameters with $P_{1}$ only [20],[21].

With the help of the derived variables $P_{1}, P_{2}$, two novel adaptive laws will be introduced in the following subsections, which are different to the gradient algorithms [12], [14], [15] and the methods using the polynomial approximation [9]-[11].

\section{A. Parameter Estimation with Constant Learning Gain}

The first adaptive estimation algorithm for updating $\hat{\alpha}$ can be given as

$$
\dot{\hat{\alpha}}(t)=-\Gamma\left(P_{1}+\kappa P_{2}\right)
$$

where $\Gamma>0$ is a constant learning gain, which can be designed as a diagonal matrix, $\kappa>0$ is a constant to balance the ability for estimating fast varying parameters and the robustness.

The convergence of adaptive law (15) is summarized as:

Theorem 1: Considering system (1) with unknown timevarying parameter $\alpha(t)$, the adaptive law (15) with derivations (2), (9), (10) and (11) is used. For the regressor $R$ being PE, then the estimation error $\tilde{\alpha}$ converges to a small compact set given by

$$
\|\tilde{\alpha}\| \leq \sqrt{\frac{\left[m \mu^{2} \gamma^{2}\left(\kappa^{2}+1 / \delta^{2}\right)+m \varpi^{2} / \lambda_{\text {min }}^{2}(\Gamma)\right] \lambda_{\max }\left(\Gamma^{-1}\right)}{2\left(\sigma_{1}-3 / 2 m\right) \lambda_{\text {min }}\left(\Gamma^{-1}\right)}} .
$$

Proof: We select a Lyapunov function as $V=\tilde{\alpha}^{T} \Gamma^{-1} \tilde{\alpha} / 2$, and then calculate the derivative of $V$ along (12), (13) and (15) as

$$
\begin{aligned}
\dot{V} & =\tilde{\alpha}^{T} \Gamma^{-1} \dot{\tilde{\alpha}}=\tilde{\alpha}^{T} \Gamma^{-1}(\dot{\alpha}-\dot{\hat{\alpha}}) \\
& =-\tilde{\alpha}^{T} G \tilde{\alpha}+\tilde{\alpha}^{T} \psi-\kappa \tilde{\alpha}^{T} R_{f}^{T} R_{f} \tilde{\alpha}+\kappa \tilde{\alpha}^{T} R_{f}^{T} \zeta+\tilde{\alpha}^{T} \Gamma^{-1} \dot{\alpha}
\end{aligned}
$$

Then by using the Young's inequality $a^{T} b \leq a^{T} a / 2 m+m b^{T} b / 2$ with a constant $m>0$, we can further obtain that

$$
\begin{aligned}
\dot{V} & \leq-\left(\sigma_{1}-3 / 2 m\right)\|\tilde{\alpha}\|^{2}+\frac{m \mu^{2} \gamma^{2}}{2 \delta^{2}}+\frac{m \mu^{2} \gamma^{2} \kappa^{2}}{2}+\frac{m \varpi^{2}}{2 \lambda_{\min }^{2}(\Gamma)} \\
& \leq-\eta V+\rho
\end{aligned}
$$

where $\eta=2\left(\sigma_{1}-3 / 2 m\right) / \lambda_{\min }\left(\Gamma^{-1}\right), \rho=m \mu^{2} \gamma^{2}\left(\kappa^{2}+1 / \delta^{2}\right) / 2$ $+m \varpi^{2} / 2 \lambda_{\text {min }}^{2}(\Gamma)$ are all positive constants for $m \geq 3 / 2 \sigma_{1}$. We can further derive the solution of (17) as $V(t) \leq e^{-\eta t} V(0)+\rho / \eta$. Based on the definition of $V=\tilde{\alpha}^{T} \Gamma^{-1} \tilde{\alpha} / 2$, one can also verify $\|\tilde{\alpha}(t)\| \leq \sqrt{2 V(t) / \lambda_{\min }\left(\Gamma^{-1}\right)} \leq \sqrt{\left(\|\tilde{\alpha}(0)\|^{2} \lambda_{\max }\left(\Gamma^{-1}\right) e^{-\eta t}+2 \rho / \eta\right) / \lambda_{\min }\left(\Gamma^{-1}\right)}$ . Therefore, the estimation error $\tilde{\alpha}(t)$ will converge to a compact set as defined in Theorem 1.

Remark 4: As proved in Theorem 1, the size of the estimation error $\tilde{\alpha}$ is affected by the excitation level (e.g., $\sigma_{1}$ ), the bound $\gamma$ of the residual error from the parameter variation $\dot{\alpha}$ and disturbance $d$, and the learning gain $\Gamma$. In general, the convergence speed $\eta$ can be increased by using a higher excitation $\sigma_{1}$ and a larger gain $\Gamma$. Moreover, it is also noted that in the specific case with constant parameters (i.e., $\dot{\alpha}=0$ ), we can verify that $\rho=0$, and thus the estimation error of the proposed adaptive algorithm (15) could converge to zero exponentially.

Remark 5: Lemma 2 indicates that the PE condition of regressor $R$ is sufficient to prove the error convergence of the proposed adaptive law (15). This condition is the same as those used in the parameter estimation literature, e.g., [9]-[12], [14], [15]. However, it is generally difficult to validate the standard $\mathrm{PE}$ condition online, which remains as an open problem. In the proposed new parameter estimation framework, we provide a feasible method to online test this condition as shown in Lemma 2, that is to calculate the minimum eigenvalue of $G$ and validate the condition $\lambda_{\min }(G)>\delta_{1}>0$.

Remark 6: For the filter constant $\delta$ in (9), a large $\delta$ can eliminate the residual error $\psi$ as shown in Lemma 3. However, a very large $\delta$ may produce a large DC gain $1 / \delta$ in (9), and then reduce the amplitude of $G$, which in turn could decrease the convergence rate of adaptive law (15). Hence, the constant $\delta$ should be set as a small constant in practice. 


\section{B. Parameter Estimation with Online Varying Learning Gain}

As shown in (12) and (13), the amplitudes of the residual error $\psi$ and the perturbing disturbance $R_{f}^{T} \zeta$ in $P_{1}$ and $P_{2}$ are related to the amplitude of the filtered regressor $R_{f}$. In this case, a constant learning gain $\Gamma$ may not be able to achieve satisfactory estimation performance for generic regressor $R$ with different amplitudes. Thus, a time-varying learning gain $Q(t)$ will be further developed to compensate for the effects of $G(t)$ and $R_{f}^{T} R_{f}$ in the adaptive law. In order to achieve this, another matrix $Q$ is defined as

$$
\dot{Q}=\delta Q-Q R_{f}^{T} R_{f} Q
$$

with non-zero initial condition $Q^{-1}(0)=Q_{0}>0$. According to the matrix equation $\frac{d}{d t} Q Q^{-1}=\dot{Q} Q^{-1}+Q \frac{d}{d t} Q^{-1}=0$, we can derive its solution as

$$
\begin{aligned}
Q(t) & =\left[e^{-\delta t} Q_{0}+\int_{0}^{t} e^{-\delta(t-\tau)} R_{f}^{T}(\tau) R_{f}(\tau) d \tau\right]^{-1} \\
& =\left[e^{-\delta t} Q_{0}+G\right]^{-1}
\end{aligned}
$$

As shown in (19), we know that $Q$ exponentially converges to $G^{-1}$, such that $G(t) Q(t) \rightarrow I$ as $t \rightarrow \infty$. Thus, it can be included in the adaptive law to eliminate the effect of $G$.

Hence, a further modified adaptive law with time-varying gain $Q$ can be designed as

$$
\dot{\hat{\alpha}}(t)=-\Gamma Q\left(P_{1}+\kappa P_{2}\right)
$$

where $\Gamma>0$ is a constant scalar, $Q$ is the matrix obtained from (18), which is used to compensate the effects of $G$ on the convergence of the adaptive law.

Before proving the convergence property of adaptive law (20), we have the following lemma:

Lemma 4: If the original regressor $R$ is PE, then the matrix $Q$ given in (18) is bounded by

$$
\gamma_{1} I \leq Q(t) \leq \gamma_{2} I
$$

where $\gamma_{1}=1 /\left(\lambda_{\min }\left(Q_{0}\right)+\mu^{2}\right)$ and $\gamma_{2}=e^{\delta T} / \varepsilon$ are positive constants.

Proof: From (19), we can rewrite its solution as $Q^{-1}(t)=e^{-\delta t} Q^{-1}(0)+\int_{0}^{t} e^{-\delta(t-\tau)} R_{f}^{T}(\tau) R_{f}(\tau) d \tau$. Considering the

PE condition of $R$, it can be verified that the inequality $Q^{-1} \geq \int_{0}^{t} e^{-\delta(t-\tau)} R_{f}^{T}(\tau) R_{f}(\tau) d \tau \geq \int_{t-T}^{t} e^{-\delta(t-\tau)} R_{f}^{T}(\tau) R_{f}(\tau) d \tau \geq e^{-\delta T} \varepsilon I$ holds for $t>T>0$. Moreover, since the filtered regressor is bounded $\left\|R_{f}\right\| \leq \mu$, we can validate that the fact $Q^{-1}(t) \leq Q^{-1}(0)+\mu^{2} \int_{0}^{t} e^{-\delta(t-\tau)} d \tau \leq Q_{0}+\mu^{2} I$ holds. Thus, the boundedness of $Q$ as given in (21) is proved.

The convergence of adaptive law (20) is described as:

Theorem 2: For system (1) with unknown time-varying parameters $\alpha(t)$, the adaptive law (20) with time-varying gain $Q$ given in (18) is used. For the regressor $R$ being PE, then the estimation error $\tilde{\alpha}$ converges to a compact set defined by $\|\tilde{\alpha}\| \leq \sqrt{\frac{\gamma_{2}\left[m \mu^{2} \gamma^{2}\left(\kappa^{2}+1 / \delta^{2}\right)+m \varpi^{2} / \gamma_{1}^{2} \Gamma^{2}\right]}{\gamma_{1}\left(2 \sigma_{1}+\delta / \gamma_{2} \Gamma-\mu^{2} / \Gamma-3 / m\right)}}$.

Proof: We select a Lyapunov function as $V=\tilde{\alpha}^{T} Q^{-1} \tilde{\alpha} / 2 \Gamma$, and then $\dot{V}$ can be calculated as

$$
\begin{aligned}
\dot{V} & =\frac{1}{\Gamma} \tilde{\alpha}^{T} Q^{-1} \dot{\tilde{\alpha}}+\frac{1}{2 \Gamma} \tilde{\alpha}^{T} \dot{Q}^{-1} \tilde{\alpha} \\
& =\frac{1}{\Gamma} \tilde{\alpha}^{T} Q^{-1} \dot{\alpha}-\tilde{\alpha}^{T} G \tilde{\alpha}+\tilde{\alpha}^{T} \psi-\kappa \tilde{\alpha}^{T} R_{f}^{T} R_{f} \hat{\alpha}+\kappa \tilde{\alpha}^{T} R_{f}^{T} \zeta \\
& +\frac{1}{2 \Gamma} \tilde{\alpha}^{T}\left(-\delta Q^{-1}+R_{f}^{T} R_{f}\right) \tilde{\alpha} \\
& \leq-\left(\sigma_{1}+\delta / 2 \gamma_{2} \Gamma-\mu^{2} / 2 \Gamma-3 / 2 m\right)\|\tilde{\alpha}\|^{2}+\frac{m \mu^{2} \gamma^{2}}{2 \delta^{2}} \\
& +\frac{m \mu^{2} \gamma^{2} \kappa^{2}}{2}+\frac{m \varpi^{2}}{2 \gamma_{1}^{2} \Gamma^{2}}
\end{aligned}
$$

where $m$ is a positive constant induced by Young's inequality. Hence, following a similar analysis given in the proof of Theorem 1, the upper bound of $\tilde{\alpha}$ (i.e., $\|\tilde{\alpha}(t)\| \leq \sqrt{2 \Gamma V \gamma_{2}}$ ) can be obtained from (22), which in turn gives the error bound given in Theorem 2. Compared to (17), it is clear shown that $\Gamma$ can be assigned large and $m$ is set small to improve the error convergence. This completes the proof.

Remark 7: It is emphasized that the adaptive laws (15) and (20) can be also adopted to estimate constant parameters (i.e., $\dot{\alpha}=0$ ) without any modification. In this case, one can verify that $\zeta=\psi=0$, and thus the adaptive law (15) can guarantee exponential convergence of estimation error to zero as shown in [20], [21].

Remark 8: The current study focuses on system (1) where the unknown parameters to be estimated are in a linearly parameterized form (i.e., $R(x, u) \alpha(t))$. However, it is potential to extend the proposed adaptive laws (15) and (20) to the cases where the unknown parameters are embedded in the nonlinear functions $F(\cdot)$ and $R(\cdot)$ possibly by applying Taylor series or min-max optimization algorithm [24], which will be studied in our future work.

Remark 9: It is shown in Theorem 1 and Theorem 2 that the estimation accuracy of the proposed algorithms depends on the varying speed of unknown parameters, i.e., $\|\dot{\alpha}\| \leq \varpi$, because the constant $\varpi$ is involved in the numerator of the upper bound of estimation error $\|\tilde{\alpha}\|$. Hence, the estimation error for fast varying parameters is larger than slowly varying parameters.

\section{COMPARISON TO OTHER ESTIMATION METHODS}

In this section, we will compare the developed adaptive law (15) with the gradient algorithm and the LS algorithm with a variable forgetting factor [3] concerning the boundedness, convergence, robustness and the required PE condition. Therefore, the error dynamics of these adaptive laws will be summarized. 


\section{A. Proposed Adaptive Law}

As it is shown in the above section, the adaptive law (15) is designed by the derived parameter estimation error. Hence, we can get its estimation error as

$$
\dot{\tilde{\alpha}}=\dot{\alpha}-\dot{\hat{\alpha}}=\dot{\alpha}-\Gamma G \tilde{\alpha}+\Gamma \psi-\kappa \Gamma R_{f}^{T} R_{f} \tilde{\alpha}+\kappa \Gamma R_{f}^{T} \zeta
$$

which indicates that $\tilde{\alpha}$ is bounded since the residual errors $\psi$, $\zeta$ and $\dot{\alpha}$ are all bounded and the error dynamics given in (23) are bounded-input-bounded-output (BIBO) stable. Moreover, as proved in Theorem 1, the estimation error approaches to a small compact set even for time-varying parameters. The bound of the estimation error is determined by the size of the residual errors $\psi$ and $\zeta$, which depends on the variation rate of the unknown parameters apart from disturbance, and thus can be small for slowly varying parameters and small disturbances.

\section{B. Gradient based Adaptive Law}

In this method, a predictor or observer has to be constructed. The observer/predictor for system (1) is given by

$$
\dot{\hat{x}}=F(x, u)+R(x, u) \hat{\alpha}+L(x-\hat{x})
$$

where $L>0$ is the observer gain, $\hat{\alpha}$ denotes the estimated parameters, which can be updated based on the following adaptive law. Define the observer error between the system state $x$ and the observer state as $e=x-\hat{x}$, then we can verify from (1) and (24) that

$$
\dot{e}=-L e+R(x, u) \tilde{\alpha}+d
$$

The gradient based adaptive law is designed to minimize the power of the estimation error $e$ by $\partial e^{2} / \partial \alpha=0$, which leads to an adaptive law by using the observer error $e$ as

$$
\dot{\hat{\alpha}}=\Gamma R^{T} \frac{e}{n^{2}}
$$

where $n^{2}$ is a normalizing factor given by $n=\sqrt{1+R^{\mathrm{T}} R}$ as explained in [14], and $\Gamma>0$ is the learning gain.

Then the estimator error of the adaptive law (26) is given as

$$
\dot{\tilde{\alpha}}=\dot{\alpha}-\dot{\hat{\alpha}}=\dot{\alpha}-\Gamma R^{T} \frac{e}{n^{2}}
$$

As it is shown in (25) and (27), although the observer error $e$ contains the information of estimation error $\tilde{\alpha}$, it is not a trivial task to calculate the bound of $\tilde{\alpha}$ in this case. In fact, the gradient based algorithm (26) $\hat{\alpha}$ can potentially result in the bursting phenomenon and thus has poor robustness against uncertainties and disturbances. Even if the observer error $e$ is bounded and small, the bound of $\tilde{\alpha}$ may be large as shown in (27). Specifically, the convergence of $\tilde{\alpha}$ could not be claimed without the PE condition even though $e$ converges to zero. In fact, compared with the LS algorithm, the gradient based algorithm can achieve faster convergence as shown in [2] and [14], whilst it is more sensitive to noise.

\section{LS Algorithm with Variable Forgetting Factor [14]}

The general idea of LS algorithm resembles that of gradient algorithm, that is, an observer error $e$ given in (24)-(25) is used to drive the adaptive law

$$
\dot{\hat{\alpha}}=H R^{T} \frac{e}{n^{2}}
$$

However, the learning gain $H$ in the LS algorithm (28) is a time-varying matrix rather than a constant gain $\Gamma$ as used in the gradient algorithm, which can be online updated by

$$
\dot{H}=-\frac{H R^{T} R H}{n^{2}}+\lambda(I-\sigma H) H
$$

where $0<I / \chi \leq H(0)=H_{0}=H_{0}^{T}<I / \sigma$ is a manually set bounded initial condition [14], and $\lambda>0$ is the forgetting factor to penalty the initial condition. Hence, the estimation error dynamics of LS algorithm can be given by

$$
\dot{\tilde{\alpha}}=\dot{\alpha}-\dot{\hat{\alpha}}=\dot{\alpha}-H R^{T} \frac{e}{n^{2}}
$$

For the LS algorithm, the estimated parameters $\hat{\alpha}$ may also drift, and the bursting phenomena may also be encountered [5]. However, as analyzed in [2], the LS method introduces an averaging effect on the error convergence, and thus can increase the robustness against noise and disturbance, while the transient convergence rate for estimating fast varying parameters may be reduced.

From above analysis, we can conclude that the proposed adaptive law (15) can guarantee that the estimation error $\tilde{\alpha}$ is bounded and converges to a small set around zero even for time-varying parameters. Moreover, the proposed estimation in this paper does not use any observer or predictor in comparison to the gradient and LS methods. Nevertheless, the robustness of the proposed adaptive law is guaranteed. Finally, with the proposed adaptive algorithm, the PE condition for guaranteeing the convergence of the estimation error can be online validated by testing the equivalent condition $\lambda_{\min }(G)>\sigma_{1}>0$.

\section{SimUlations}

In this section, the proposed estimation methods are validated by conducting simulations based on a benchmark servo motor system. The gradient algorithm and the LS algorithm with variable forgetting factor given in the above section are also simulated for comparison.

The following servo motor system [7], [10] is used in this section as the simulation plant

$$
\left\{\begin{array}{l}
\dot{x}_{1}=x_{2} \\
\dot{x}_{2}=\underset{F(x, u)}{10 u}+\underbrace{\left[\begin{array}{ll}
-x_{2} & -10 \arctan \left(900 x_{2}\right)
\end{array}\right]}_{R(x, u)} \underbrace{\left[\begin{array}{l}
\alpha_{1}(t) \\
\alpha_{2}(t)
\end{array}\right]}_{\alpha(t)}+d
\end{array}\right.
$$

where $\quad \alpha_{1}(t)=2+\sin (t) \quad$ and $\quad \alpha_{2}(t)=3+\cos (0.5 \pi t) \quad$ are the unknown time-varying parameters to be estimated; $u(t)=1000 \sin (20 \pi t)$ is the control input. To validate the robustness of the proposed algorithms, a zero-mean random signal $d(t)=1 \cdot \operatorname{rand}(1)-0.5$ is added into the system, which can be taken as the measurement noise of the velocity sensor. The adaptive parameter estimation methods presented in Section III are tested in order to investigate the efficacy of the intermediate variables $Q, P_{1}$ and $P_{2}$. The parameters used in 
(2) and (9) are set as $k=0.001, \delta=70$. We first simulate the adaptive law (15) with the constant learning gain $\Gamma=\operatorname{diag}\left(\left[\begin{array}{ll}5 & 400\end{array}\right]\right)$ and the zero initial condition $\hat{\alpha}(0)=0$.
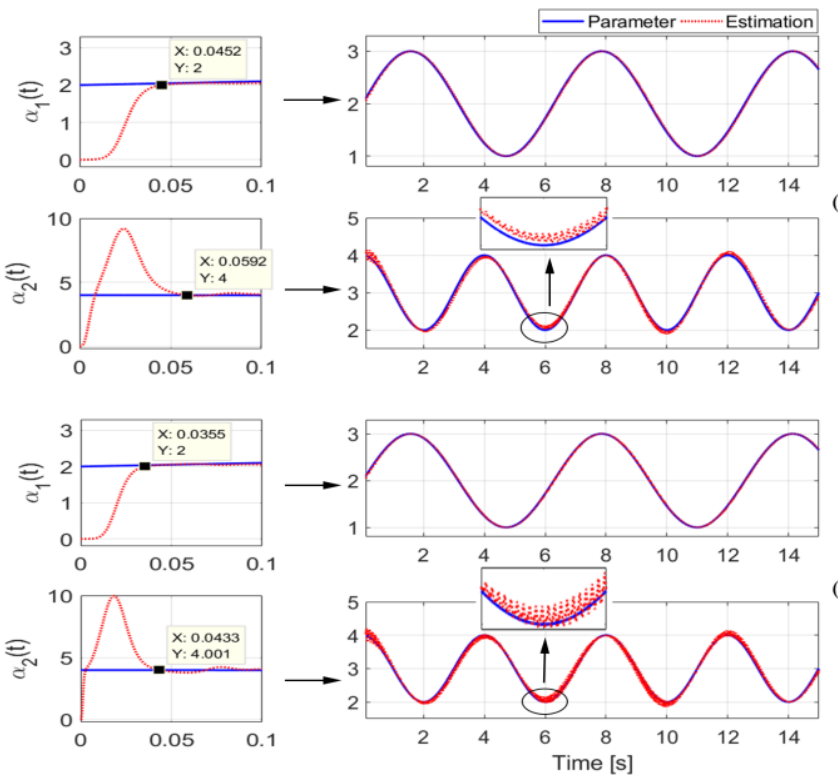

(b)

Fig. 1 Estimated parameters of adaptive law (15): (a) $\kappa=0$; (b) $\kappa=0.03$.
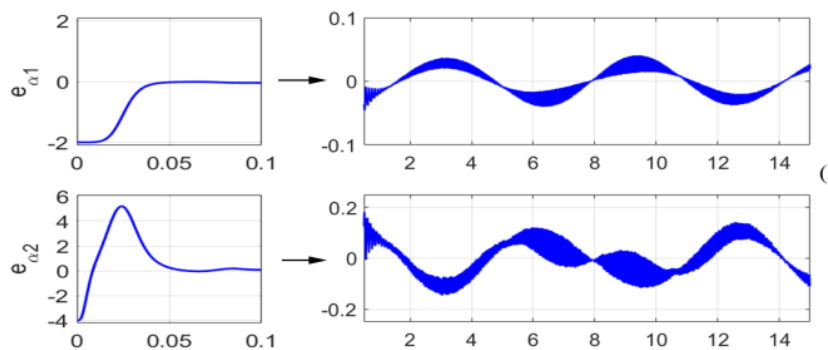

(a)
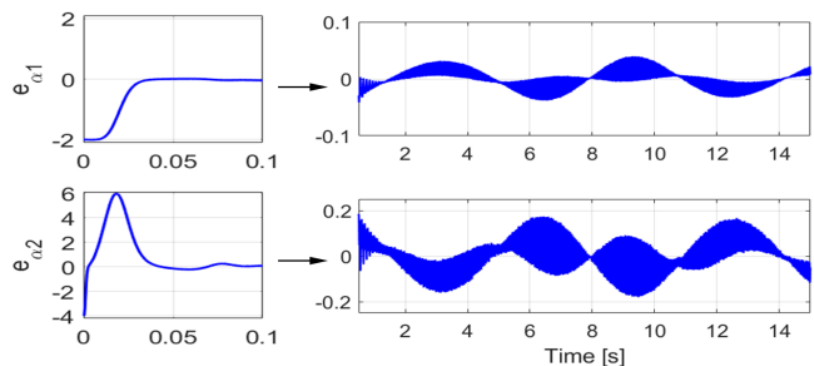

Fig. 2 Estimation errors of adaptive law (15): (a) $\kappa=0$; (b)

$$
\kappa=0.03 \text {. }
$$

Simulation results of the estimated parameter profiles and the corresponding estimation errors are depicted in Fig. 1 and Fig. 2. It is shown that adaptive law (15) with both $\kappa=0$ and $\kappa=0.03$ can achieve accurate estimation of the unknown time-varying parameters in the steady-state. However, the estimator with $P_{2}$ of instant estimation error $R_{f}^{T} R_{f} \tilde{\alpha}$ (i.e., $\kappa=0.03$ ) can obtain slightly faster transient convergence performance than that without $P_{2}$ (i.e., $\kappa=0$ ). In the case $\kappa=0.03$, the instant error information in $P_{2}$ is used together with $P_{1}$, which can help tracking time-varying parameters. However, the use of instant information $R_{f}^{T} R_{f} \tilde{\alpha}$ in the adaptive law makes it sensitivity to noise, which can result in more high-frequency oscillations as shown in Fig. 1 and Fig. 2. Hence, in practice we cannot set $\kappa$ as a large constant.
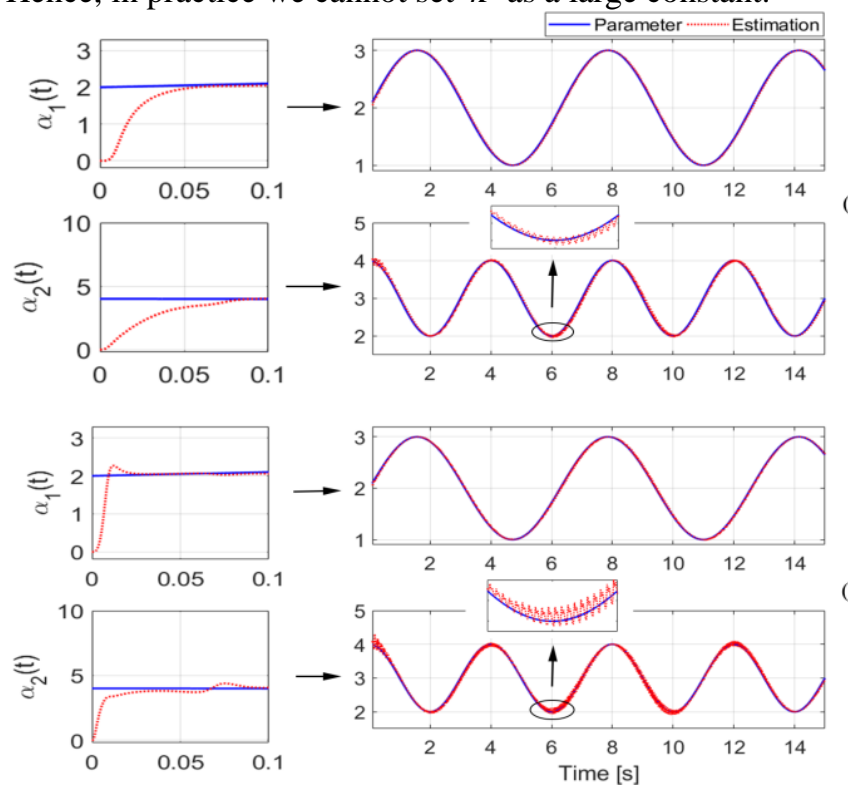

(b)

Fig. 3 Estimated parameters of adaptive law (20): (a) $\kappa=0$; (b) $\kappa=0.03$.

We further test adaptive law (20), where a time-varying gain $Q$ given in (18) is used. The parameters of $k$ and $\delta$ are the same as in the previous case. However, as analyzed in Section III, the effects of regressor $R$ on the convergence rate can be eliminated by using time-varying gain $Q$ and thus the learning gain of the adaptive law (20) is retuned as $\Gamma=\operatorname{diag}\left(\left[\begin{array}{ll}70 & 55\end{array}\right]\right)$ for both $\kappa=0$ and $\kappa=0.03$. As shown in Fig. 3 and Fig. 4 , the estimator (20) with a time-varying learning gain $Q$ can retain better performance than that with a constant learning gain (e.g., (15)), that is adaptive law (20) achieves smaller, smoother transient estimation errors than adaptive law (15), in particular the overshoot during the first few seconds can be significantly eliminated, since the adopted $Q$ can compensate for the influence of matrix $G$ in the transient stage. Moreover, both the adaptive laws (15) and (20) with $\kappa=0.03$ as shown in Fig. 1(b) and Fig. 3 (b) indicates that the use of $P_{2}$ can achieve fast transient convergence response.

To evaluate the estimation performance of the proposed estimation methods quantitatively, the estimation errors of the unknown parameter $\alpha_{2}(t)=3+\cos (0.5 \pi t)$ are utilized in the following statistical evaluation.

1) Integrated Squared Error (ISE)

$$
\mathrm{ISE}=\int e^{2} d t
$$

2) Maximum Absolute Error (MAE)

$$
\mathrm{MAE}=\max (|e|)
$$

3) Standard Deviation (SD)

$$
\mathrm{SD}=\sqrt{\frac{1}{T} \int\left[e-\mu_{e}\right]^{2} d t}
$$

with $\mu_{e}$ being the average estimation error. The ISE describes 
the overall estimation error response. The MAE evaluates the temporal difference between the true value and the estimates. The SD can quantify the estimation error variation.
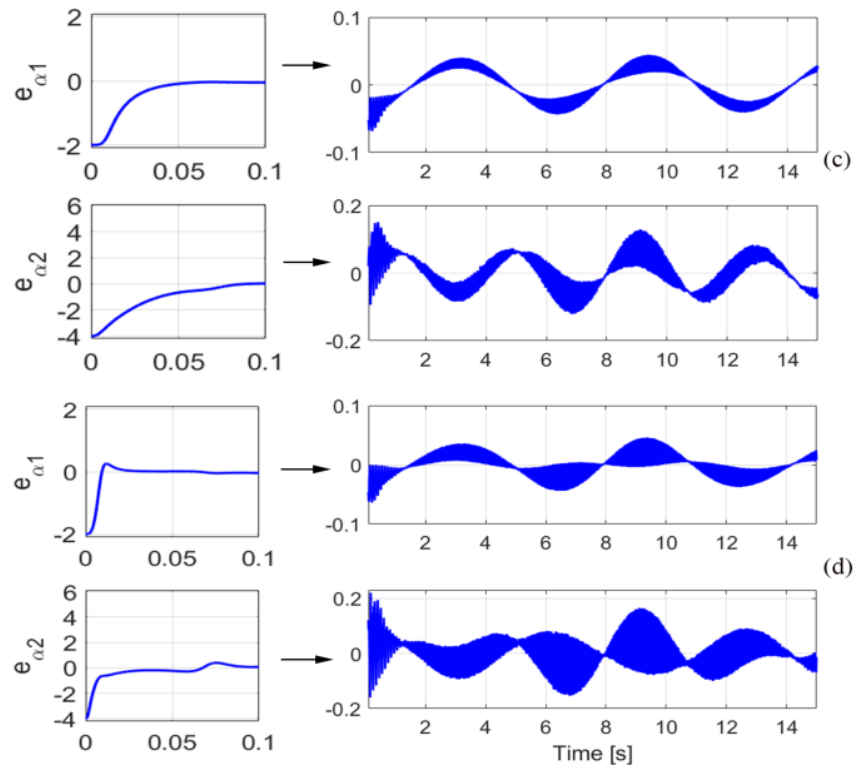

(d)

Fig. 4 Estimation errors of adaptive law (20) with: (c) $\kappa=0$; (d) $\kappa=0.03$.

These three performance indices of the above four different adaptive laws are calculated based on the collected data between $4 \mathrm{~s}$ to $15 \mathrm{~s}$, and shown in Table I. It is observed that the adaptive law (20) using time-varying gains has improved response than adaptive law (15) using constant learning gains. Specifically, the ISE and SD of (20) with $\kappa=0.03$ are smaller than the other cases. Moreover, it can be found that the MAE of (15) is slightly larger than that of (20). From the aforementioned results, we know that the proposed algorithm (20) with a time-varying gain $Q$ and instant error information $P_{2}$ performs superior over other tested algorithms.

\begin{tabular}{ccccc}
\multicolumn{3}{c}{ TABLE I. ESTIMATION PERFORMANCE FOR $\alpha_{2}$} \\
\hline \hline Indices & \multicolumn{2}{c}{ Constant Gain } & \multicolumn{2}{c}{$\begin{array}{c}\text { Time-varying } \\
\text { Gain }\end{array}$} \\
\hline$\kappa$ & 0 & 0.03 & 0 & 0.03 \\
\hline ISE & 0.0365 & 0.0398 & 0.0266 & 0.0247 \\
MAE & 0.1425 & 0.1793 & 0.1282 & 0.1540 \\
SD & 0.0937 & 0.0989 & 0.0814 & 0.0805 \\
\hline \hline
\end{tabular}

To further reveal the merit of adaptive approaches using the estimation error, the proposed method (20) with a time-varying gain $Q$ and $P_{2}$ is compared with the gradient algorithm (26) and the LS algorithm (28) with a forgetting factor (29) elaborated in Section IV. For fair comparison, the initial condition is set as the same for all three methods, that is $\hat{\alpha}(0)=0$. The random signal $d(t)=1 \cdot \operatorname{rand}(1)-0.5$ is also used to test the robustness. The parameters used in the gradient algorithm (25) and (26) are $L=10^{2}, \Gamma=10^{4} \cdot \operatorname{diag}\left(\left[\begin{array}{ll}2 & 10\end{array}\right]\right)$. The parameters in the LS method (25), (28) and (29) are chosen as $L=10^{2}, \lambda=10^{5}$, $\chi=1$ and $\sigma=10^{-7}$. Fig. 5 illustrates the parameter estimation results of the gradient method (26) and LS method (28), respectively. As it can be seen from Fig. 5, the time-varying parameters cannot be estimated accurately by using the gradient method (26) and LS method (28). The estimation errors of these two algorithms shown in Fig. 5 are clearly larger than that shown in Fig. 2 and 4 with the proposed algorithms (15) and (20). This has been explained in the above analysis, i.e., the proposed adaptive laws (15) and (20) are derived based on the extracted estimation error rather than the observer error as shown in Fig. 6. Moreover, we can find from Fig. 5 that the LS approach performs slightly better than the gradient algorithm in terms of steady-state estimation error and smoothness of the estimated parameters, which is attributed to the induced filter effect as analyzed in Section IV, though this leads to a small phase lag in the estimated parameters indicated in Fig. 5. On the other hand, the gradient method is more sensitive to noise, which can be viewed in both Fig. 5 and Fig. 6 .
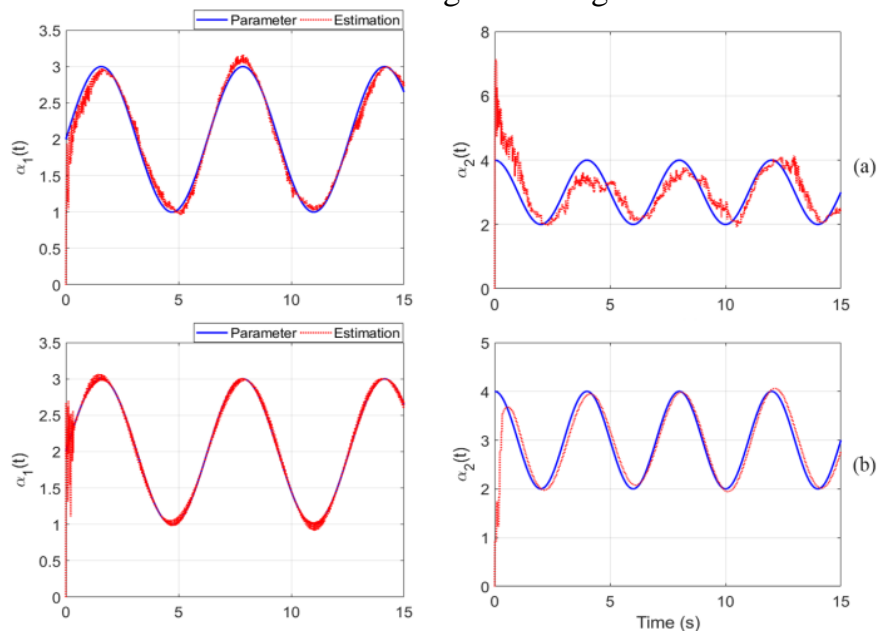

Fig. 5 Parameter estimation: (a) gradient adaptive law (26); (b) LS adaptive law (28).
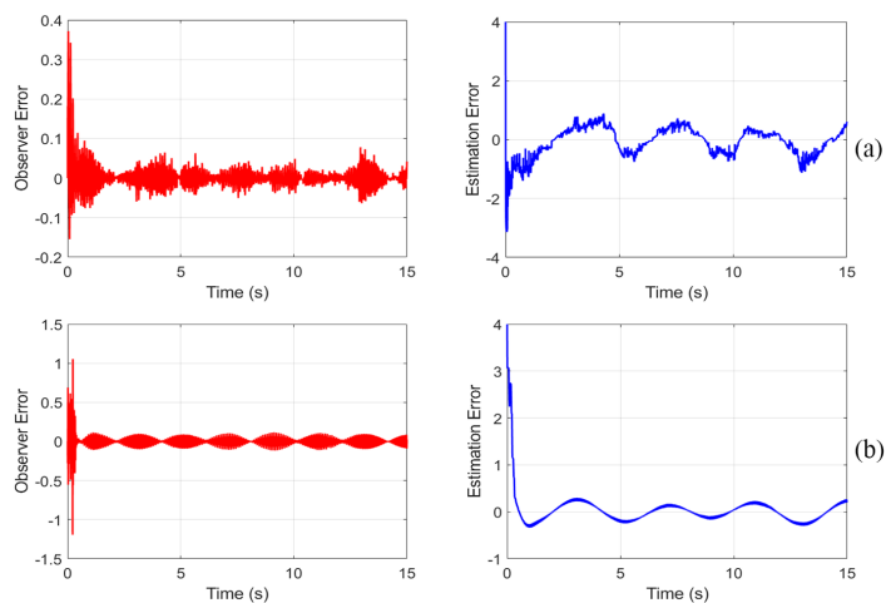

Fig. 6 Observer errors for $x_{2}$ and estimation errors for $\alpha_{2}$ : (a) gradient adaptive law (26); (b) LS adaptive law (28).

Finally, to test the ability of the proposed adaptive laws for tracking complex, fast varying parameters, we set the unknown parameters of system (31) as multi-frequency sinusoidal signals: $\alpha_{1}(t)=2+\sin (t)+\cos (3 t)$ and $\alpha_{2}(t)=3+\cos (0.5 \pi t)+\sin (\pi t)$. The proposed adaptive law (20) and the LS algorithm (28) with 
a forgetting factor (29) are all implemented. Simulation results of the estimated parameter profiles and the corresponding estimation errors are depicted in Fig. 7 and Fig. 8. It is shown that the proposed adaptive law (20) can retain better estimation performance than the LS method (28) even for multi-frequency parameters, i.e., the estimation errors of the proposed method are smaller than that of LS algorithm.
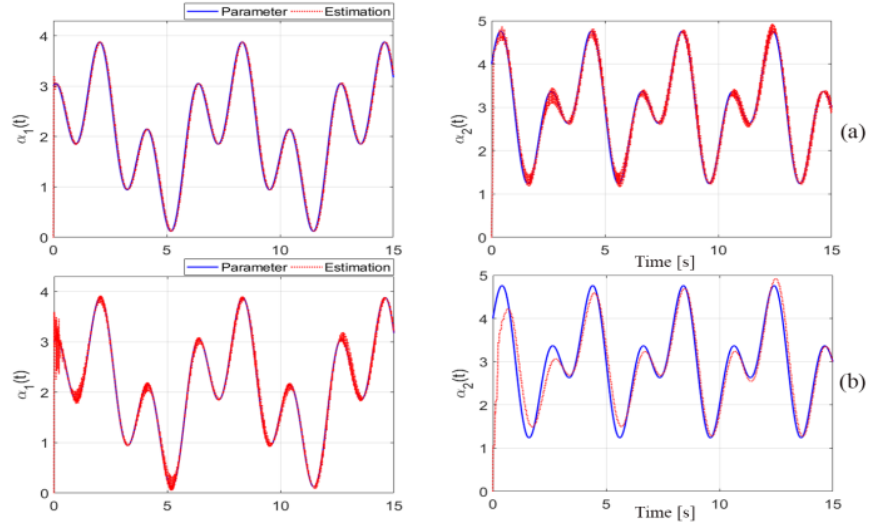

Fig. 7 Estimation results for unknown multi-frequency parameters: (a) adaptive law (20); (b) LS adaptive law (28).
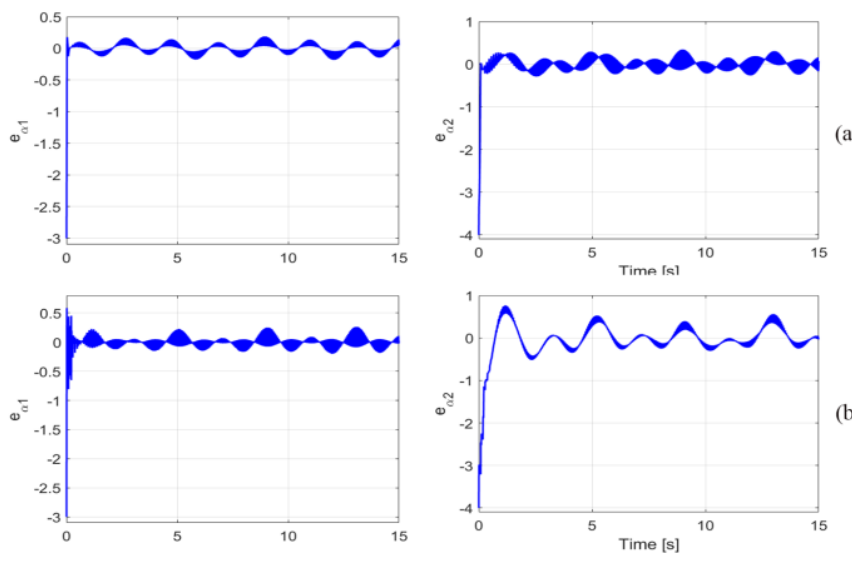

Fig. 8 Estimation errors for unknown multi-frequency parameters: (a) adaptive law (20); (b) LS adaptive law (28).

\section{PRACTICAL EXPERIMENTS}

This section will validate the proposed estimation approaches by conducting experiments based on a laboratory roto-magnet plant.

\section{A. Description of Roto-Magnet Plant}

The roto-magnet plant built in our laboratory is used as a control plant for educational purpose [25]. This plant is a mechatronic device, composed of a DC motor, a rotational bar, a permanent magnet and two fixed electromagnets. More specifically, the permanent magnet with two opposite magnetic poles is supported by a rotational bar. Two electromagnets with different magnetic poles are fixed near the bar, as shown in Fig. 9. A small DC motor under the experimental table is attached to another side of the bar.

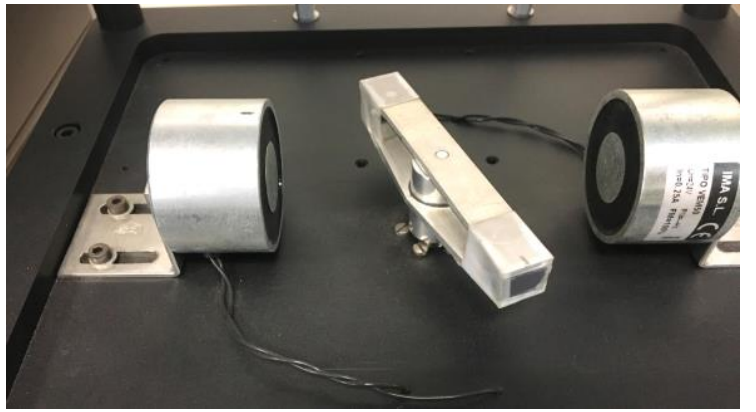

Fig. 9 Structure of the roto-magnet plant

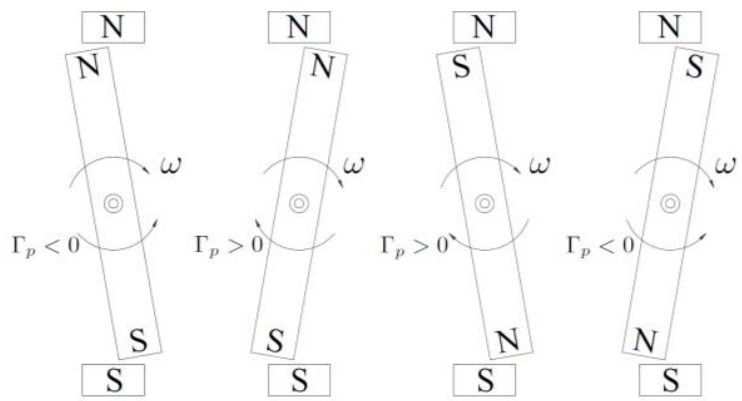

Fig. 10 Schematic of the interation between fixed eletromagnets and a moving permanent magnet [25].

During the operation of DC motor, the interaction between the fixed electromagnets and a moving magnet creates a magnetic field that causes a pulsating load torque $\Gamma_{p}$ on the movement of the bar. The pulsating load torque $\Gamma_{p}$ is considered as a disturbance in this system, since it affects dynamic behaviors of the control output response.

Since the magnetic field depends on the relative position between different magnets, the period of the torque acting over the bar is $2 \pi$ (as seen in Fig. 10). The relative position between different magnets is determined by the rotational angle $\theta$. The pulsating load torque $\Gamma_{p}$ also depends on the rotational angle $\theta$. Hence, under a constant angular speed (i.e., $\ddot{\theta}=\dot{\omega}=0$ ), the pulsating load torque $\Gamma_{p}$ becomes a periodic signal, where its fundamental period mainly depends on this constant rotational speed. Nevertheless, it is very hard to measure this pulsating load torque in the system. In order to eliminate the effect of this disturbance and control the plant properly, some parameters associated to this torque generator need to be estimated.

The model of this plant can be described as

$$
\left\{\begin{array}{l}
\dot{\theta}=\omega \\
\dot{\omega}=-\alpha_{1}(t) \omega+\Gamma_{p}(\theta)+\alpha_{2}(t) u
\end{array}\right.
$$

where $\alpha_{1}(t)$ is the time-varying friction coefficient, $\alpha_{2}(t)$ is the input gain, and $u$ is the control action, and the pulsating load torque can be defined as $\Gamma_{p}(\theta)=\sum_{k_{1}=1}^{N}\left[\delta_{k_{1}} \sin \left(k_{1} \theta\right)+\gamma_{k_{1}} \cos \left(k_{1} \theta\right)\right]$.

Hence, to obtain a precise model of this plant, the unknown, possibly time-varying parameters (e.g., $\alpha_{1}, \alpha_{2}, \delta_{k 1}$ and $\gamma_{k 1}$ ) should be estimated by using the measured input and output variables (e.g., $u, \omega$ and $\theta$ ). 


\section{B. Experiment Configuration}

To facilitate the estimation with measured input $u$, angular velocity $\omega$ and angle $\theta$, the experimental platform consists of the roto-magnet plant, an actuator, a speed sensor, a PC controller and some signal converters. The DC motor (Johnson Electric HC615L) is the main component in the actuator. The DC motor produces a rotation speed $\omega$ and its corresponding motor torque acting on the roto-magnet plant. The speed sensor measures the angular speed on the moving magnet. The collected input/output variables used for parameter estimation are shown in Fig. 11.
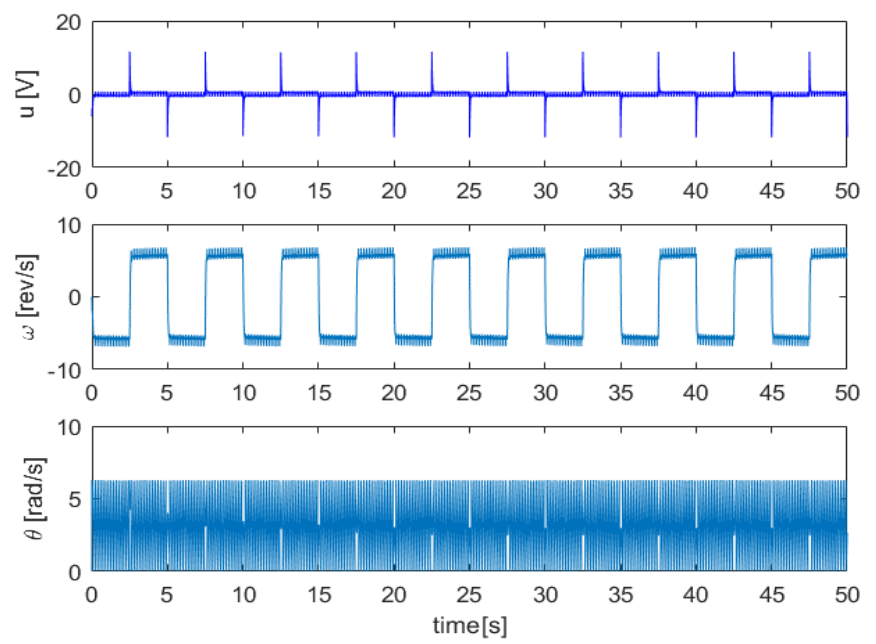

Fig. 11 The measured variables of the test plant.

\section{Practical Results}

A higher order $N$ of the load torque generation model in (35) could help to describe more accurate physical torque generation dynamics. However, a large model order $N$ will increase the computational costs of the estimation since the dimension of regressor will be increased. In order to seek a tradeoff between the model accuracy and the computational costs, we set the order parameter as $N=1$, such that the load torque can be given as $\Gamma_{p}(\theta)=\delta_{1} \sin (\theta)+\gamma_{1} \cos (\theta)+d_{1}$, where $d_{1}$ denotes the effects of the residual modeling error, which can be estimated together with $\alpha_{1}, \alpha_{2}, \delta_{1}$ and $\gamma_{1}$. In the experiments, the parameters used in the adaptive law (15) are: $k=0.01, \delta=1, \kappa=0.01$ and $\Gamma=1 \cdot \operatorname{diag}\left(\left[\begin{array}{lllll}34 & 40 & 25 & 3 & 3\end{array}\right]\right)$. Fig. 12 depicts the results of the parameter estimation. It can be found from Fig. 12 that the estimated parameters are fast varying corresponding to the rotation velocity $\omega$ and angle $\theta$. In particular, the friction coefficient $\alpha_{1}$ varies along with rotation velocity $\omega$, and the input gain $\alpha_{2}$ is almost a constant around 1.67 though there are minor oscillations.

In order to verify the estimation results, we use the estimated parameters and the control input $u$ to reconstruct the plant model (35), and then compare the rotation angle output of the derived model with the measured output data $\theta$. The comparative results are shown in Fig. 13. From Fig. 13, we can find that the model output $\theta$ fits the c measured system output $\theta$ very well, which implies that the estimated parameters given in Fig. 12 can capture the system dynamics effectively.

Finally, the LS algorithm (28) with a forgetting factor (29) is used to estimate the parameters by using the same plant model (35). Estimation results and the corresponding outputs between the model and the measured system output are depicted in Fig. 14 and Fig. 15, respectively. Comparing Fig. 12 with Fig. 14, it is found that the estimated parameters based on the LS algorithm (28) tend to be constants, which illustrate the limited ability of LS algorithm to track fast varying parameters in the system. This will influence the accuracy of the derived model with these estimated parameters. Hence, as shown in Fig.15, the model output $\theta$ cannot match the collected system output $\theta$ well. This comparative result again shows that the proposed adaptive law (20) can retain better estimation performance than the LS method (28).
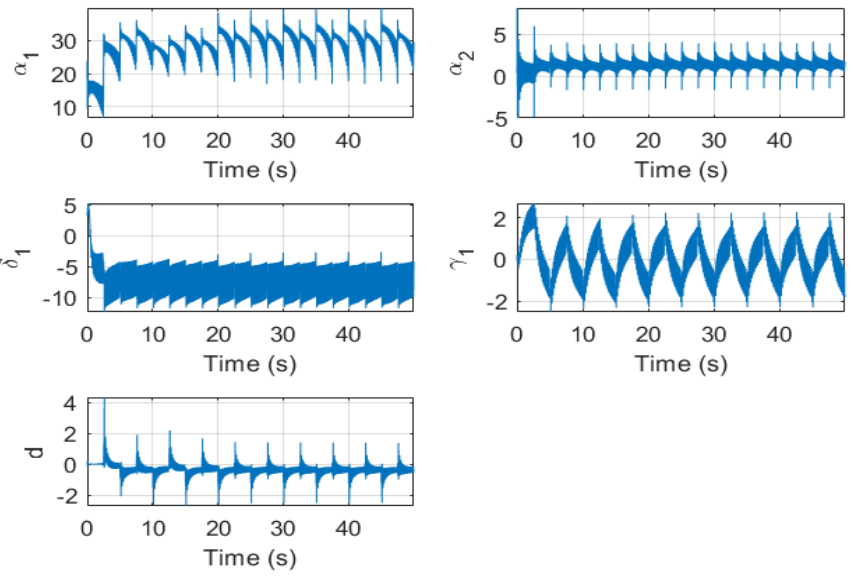

Fig. 12 Estimated parameters for roto-magnet plant with adaptive law (20).

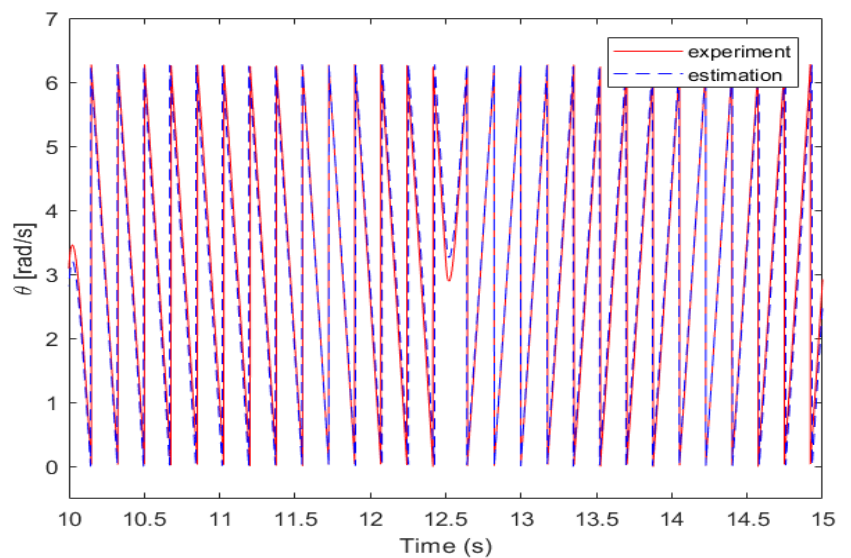

Fig. 13 Comparison between the model output with adaptive law (20) and the measured plant output. 

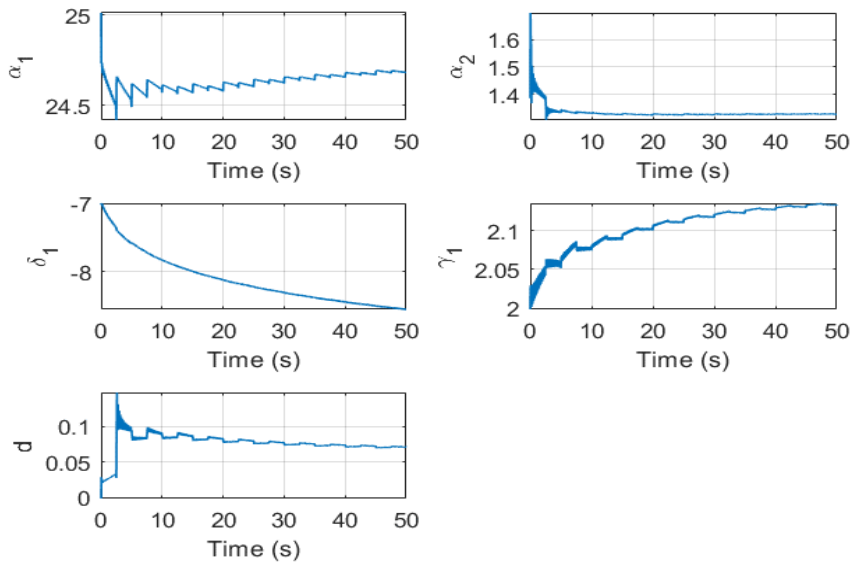

Fig. 14 Estimated parameters for roto-magnet plant with LS adaptive law (28).

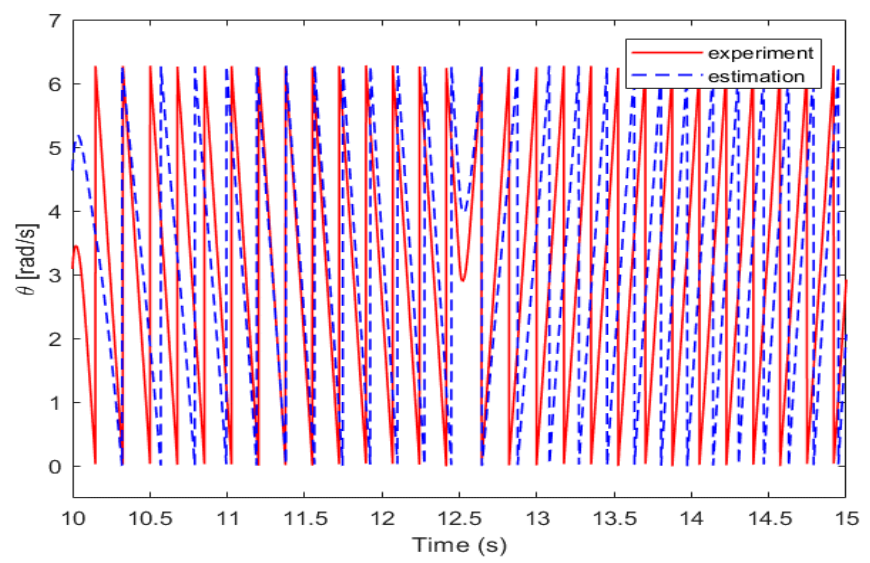

Fig. 15 Comparison between the model output with LS adaptive law (28) and the measure plant output.

\section{CONCLUSION}

In this paper, we introduce a new adaptive parameter estimation approach for nonlinear systems with time-varying parameters. The developed adaptive laws are designed based on the extracted parameter estimation errors, and thus do not require any observer/predictor design and the measurements of the derivative of system states. Moreover, this new estimation framework provides a feasible method to online test the required $\mathrm{PE}$ condition to guarantee the error convergence. A time-varying gain is further introduced to eliminate the effect of the regressor to achieve better convergence and robustness. The convergence of the estimation error has been proved in terms of Lyapunov method. Comparative simulation results illustrate that the suggested approaches can achieve better performance than the gradient based algorithm and LS algorithm. Practical experiments based on a roto-magnet plant are also conducted to show the applicability of these methods. Future works will focus on extending the proposed estimation approaches to systems where the unknown parameters are involved in the nonlinear functions.

\section{REFERENCES}

[1] S. Sastry, M. Bodson, and J. F. Bartram, Adaptive Control: Stability, Convergence, and Robustness. Prentice Hall, 1989.
[2] J.-J. E. Slotine and W. Li, Applied Nonlinear Control. Prentice Hall, 1991.

[3] K. S. Narendra and A. M. Annaswamy, Stable adaptive systems. Prentice Hall, 1989.

[4] B. D. O. Anderson and A. Dehghani, "Challenges of adaptive control-past, permanent and future," Annu. Rev. Control, vol. 32, no. 2, pp. 123-135, Dec. 2008.

[5] P. A. Ioannou and J. Sun, Robust adaptive control, vol. 1. PTR Prentice-Hall Upper Saddle River, NJ, 1996.

[6] J. Na, G. Herrmann, R. Burke, and C. Brace, "Adaptive input and parameter estimation with application to engine torque estimation," in 2015 54th IEEE Conference on Decision and Control (CDC), 2015, pp. 3687-3692.

[7] S. Dhaliwal and M. Guay, "Set-based adaptive estimation for a class of nonlinear systems with time-varying parameters," IFAC Proc. Vol., vol. 45, no. 15, pp. 391-395, Jan. 2012.

[8] J. Na, "Adaptive prescribed performance control of nonlinear systems with unknown dead zone," Int. J. Adapt. Control Signal Process., vol. 27, no. 5, pp. 426-446, 2013.

[9] Y. Zhu and P. R. Pagilla, "Adaptive Estimation of Time-Varying Parameters in Linearly Parametrized Systems," J. Dyn. Syst. Meas. Control, vol. 128, no. 3, pp. 691-695, 2006.

[10] J. Chen, G. Zhang, and Z. Li, "On-line parameter estimation for a class of time-varying continuous systems with bounded disturbances," Int. J. Adapt. Control Signal Process., vol. 25, no. 1, pp. 18-32, Jan. 2011.

[11] J. Na, J. Yang, X. Ren, and Y. Guo, "Robust adaptive estimation of nonlinear system with time-varying parameters," Int. J. Adapt. Control Signal Process., vol. 29, no. 8, pp. 1055-1072, 2015.

[12] R. H. Middleton and G. C. Goodwin, "Adaptive control of time-varying linear systems," IEEE Trans. Autom. Control, vol. 33, no. 2, pp. 150-155, Feb. 1988.

[13] F. Ding, P. X. Liu, and H. Yang, "Parameter Identification and Intersample Output Estimation for Dual-Rate Systems," IEEE Trans. Syst. Man Cybern. - Part Syst. Hum., vol. 38, no. 4, pp. 966-975, Jul. 2008.

[14] M. de Mathelin and R. Lozano, "Robust adaptive identification of slowly time-varying parameters with bounded disturbances," Automatica, vol. 35, no. 7, pp. 1291-1305, Jul. 1999.

[15] R. Lozano, D. Dimogianopoulos, and R. Mahony, "Identification of linear time-varying systems using a modified least-squares algorithm," Automatica, vol. 36, no. 7, pp. 1009-1015, Jul. 2000.

[16] F. Ding, L. Xu, and Q. Zhu, "Performance analysis of the generalised projection identification for time-varying systems," IET Control Theory Amp Appl., vol. 10, no. 18, pp. 2506-2514, Sep. 2016.

[17] E. Moshksar and M. Guay, "Almost invariant manifold approach for adaptive estimation of periodic and aperiodic unknown timevarying parameters," Int. J. Adapt. Control Signal Process., vol. 30, no. 1, pp. 76-92, 2016.

[18] X. Yang, S. Yin, and O. Kaynak, "Robust Identification of LPV Time-Delay System With Randomly Missing Measurements," IEEE Trans. Syst. Man Cybern. Syst., pp. 1-11, 2018.

[19] F. Guo, O. Wu, H. Kodamana, Y. Ding, and B. Huang, "An Augmented Model Approach for Identification of Nonlinear Errors-in-Variables Systems Using the EM Algorithm," IEEE Trans. Syst. Man Cybern. Syst., pp. 1-11, 2017.

[20] J. Na, M. N. Mahyuddin, G. Herrmann, X. Ren, and P. Barber, "Robust adaptive finite-time parameter estimation and control for robotic systems," Int. J. Robust Nonlinear Control, vol. 25, no. 16, pp. 3045-3071, 2015.

[21] J. Na, M. N. Mahyuddin, G. Herrmann, and X. Ren, "Robust adaptive finite-time parameter estimation for linearly parameterized nonlinear systems," in Proceedings of the 32nd Chinese Control Conference, 2013, pp. 1735-1741. 
[22] J. Na, A. S. Chen, G. Herrmann, R. Burke, and C. Brace, "Vehicle Engine Torque Estimation via Unknown Input Observer and Adaptive Parameter Estimation," IEEE Trans. Veh. Technol., vol. 67, no. 1, pp. 409-422, Jan. 2018.

[23] A. Astolfi and R. Ortega, "Immersion and invariance: a new tool for stabilization and adaptive control of nonlinear systems," IEEE Trans. Autom. Control, vol. 48, no. 4, pp. 590-606, Apr. 2003.

[24] C. Cao, A. M. Annaswamy, and A. Kojic, "Parameter convergence in nonlinearly parameterized systems," IEEE Trans. Autom. Control, vol. 48, no. 3, pp. 397-412, Mar. 2003.

[25] R. Costa-Castelló, J. Nebot, and R. Grinó, "Demonstration of the internal model principle by digital repetitive control of an educational laboratory plant," IEEE Trans. Educ., vol. 48, no. 1, pp. 73-80, 2005.

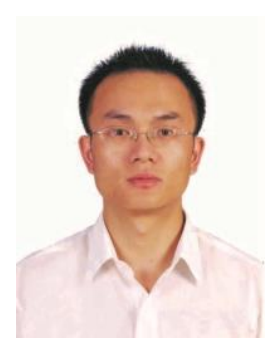

Jing Na (M'15) received the B.Sc. and Ph.D. degrees from the School of Automation, Beijing Institute of Technology, Beijing, China, in 2004 and 2010, respectively.

From 2011 to 2013, he was a Monaco/ITER Postdoctoral Fellow at the ITER Organization, Saint-Paul-lès-Durance, France. From 2015 to 2017, he was a Marie Curie Intra-European Fellow with the Department of Mechanical Engineering, University of Bristol, U.K. Since 2010, he has been with the Faculty of Mechanical and Electrical Engineering, Kunming University of Science and Technology, Kunming, China, where he became a Professor in 2013. He has coauthored one monograph published in Elsevier and authored or coauthored over 100 international journal and conference papers. His current research interests include intelligent control, adaptive parameter estimation, nonlinear control and applications for robotics, vehicle systems, and wave energy convertor.

Dr. Na was a recipient of the Marie Curie Fellowship from EU, the Best Application Paper Award of the 3rd IFAC International Conference on Intelligent Control and Automation Science in 2013, and the 2017 Hsue-shen Tsien Paper Award. $\mathrm{He}$ is currently an Associate Editor of Neurocomputing and has served as the International Program Committee Chair of ICMIC in 2017.

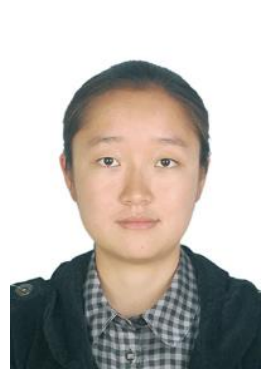

Yashan Xing was born in Yunnan, China. She received the B.Sc. degree in mechanical engineering from Kunming University of Science and Technology, Yunnan, China, in 2014. In 2017, she received the M.Sc. degrees in mechanical engineering from both Blekinge Tekniska Högskola, Blekinge, Sweden and Kunming University of Science and Technology, Yunnan, China. She is currently pursuing the $\mathrm{Ph} . \mathrm{D}$. degree in control engineering from the Universitat Politècnica de Catalunya, Barcelona, Spain.

Her current research interests include modelling, adaptive control and parameter estimation for solid oxide fuel cells and solid oxide electrolysis cells.

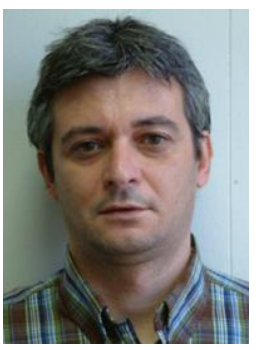

Ramon Costa-Castelló (M'94-SM'07) was born in Lleida, Catalunya, Spain in 1970. He obtained the M.Sc. degree in computer science in 1993 from the Facultat d'Informàtica de Barcelona (FIB), the Universitat Politècnica de Catalunya (UPC). In 2001 he received the Ph.D. degree in computer science from the Advanced Automation and Robotics (AAR) program from the Cibernetics Institute (Institut de Cibernètica, IC) at UPC.

Currently, he is an Associate Professor at the Automatic Control department (Department of Enginyeria de Sistemes Automàtica i Informàtica Industrial, ESAII) from UPC and the Institut de Robòtica i Informàtica Industrial (a Joint Research Center of the Spanish Council for Scientific Research (CSIC) and UPC).

$\mathrm{He}$ is a Senior Member from the Institute of Electrical and Electronics Engineers (IEEE), member of the Comité Español de Automática (CEA) and member of IFAC (EDCOM, TC 9.4 Committee). 